\title{
OPEN-HEREDEUX: OPEN HEuristic REsource for Designing and Evaluating User eXperience
}

\author{
Llúcia Masip, Marta Oliva, and Toni Granollers \\ Department of Computer Science and Industrial Engineering, \\ University of Lleida (Spain) \\ \{lluciamaar, oliva, tonig\} @diei.udl.cat
}

\begin{abstract}
The need of enhancing design and evaluation of user experience exists. We propose a resource which helps to semi automate the design and evaluation of user experience.
\end{abstract}

Keywords: User experience, evaluation resources, heuristic evaluation.

\section{User Experience}

The concept of User eXperience (UX) includes more facets than traditional ones related to usability. According to Peter Morville [1], UX includes useful, usable, valuable, desirable, findable, credible and accessible. Moreover, other authors also consider aspects such as communicability, cross-culture, emotions or playability.

Facets help us to improve the quality of use in interactive systems and, consequently, to increase UX. Despite the new tendency in UX, currently there is no specific technique to evaluate UX when users interact with an Interactive System (IS) [2]. Certainly, UX experts can use usability evaluation methods for evaluating UX, because the "oldest" and the most traditional facet used when evaluating aspects related to the quality of use is usability. Nevertheless, a consensus about what is the best methodology to evaluate UX still does not exist [2].

The project which I am involved in with my $\mathrm{PhD}$ is contextualized in UX evaluation but focusing our efforts on exploiting the capabilities of one of the methods used most: the heuristic evaluation (HE). HE, as it is known in HCI field, was spread by Nielsen and Molich [3] but HE had been discreetly created by Ravden and Johnson in [4]. It is one of the most challenged and successful methods for evaluating the usability of interactive systems. Nevertheless, HE presents barriers that induce a slower and more expensive evaluation process that could lessen all its advantages. The first deficiency is that the whole evaluation process is based on considered heuristics. A review of this set, to adapt it to specific features of interactive systems is always essential despite the fact that experts have done many efforts to provide a whole set of heuristics and apply them to different interactive systems. The second deficiency is in the evaluation result. Usually, the method provides qualitative results. And more often than not, qualitative results are not enough if these results do not allow us to take decisions in an objective way like it happens with quantitative results. In this context, the following sections explain our ideas to go a step beyond to provide solutions for the mentioned above problems. 


\section{OPEN-HEREDEUX}

OPEN-HEREDEUX is the short name which we use to refer to the project called: "OPEN HEuristic Resource for Designing and Evaluating User eXperience in interactive systems". It will enable UX experts to design and evaluate UX in a semi automates way. Its four components are detailed below.

\subsection{Open Repository of Heuristics}

The main goal of this component is to manage information. We should provide a repository with all the information needed to achieve a complete and minimum set of heuristics and to consider all aspects of UX either in its design phase or evaluation process for a specific IS.

Therefore, the repository will have all information such as a whole IS classification [5], essential facets to evaluate UX in any IS and a complete set of heuristics related to all the facets mentioned previously.

Once we have all this information, next step will be data modeling; we will model information through ontologies. Finally, all information will be stored with the most convenient technique to be able to extend, modify or delete this information later. All this information will be used as input for the next component, the heuristics adviser.

\subsection{Adviser of Heuristics}

The main goal of the heuristics adviser component is to propose the most convenient list of heuristics to be used (for the specific system to be analyzed), such as recommendation principles in a design phase or such as evaluation principles in a UX evaluation based on heuristics.

The adviser considers for its suggestion different factors according to the type of IS and other factors such as economics, etc. About the type of IS the adviser should at least regard the facets considered in this IS and the minimum but complete set of heuristics of each facet according to the user profile that needs these heuristics (designer or evaluator). In reference to economical factors, the heuristics adviser should consider the time that designers or evaluators have to take into account for the set of heuristics and the money invested in the project. These factors should determine the minimum number of heuristics that the adviser should suggest. This component has an important advantage when choosing the best heuristics for a specific IS: turning a manual process and process based on UX expert experience to semi automate process where UX experts will only review the set of heuristics suggested.

Finally, heuristic suggestions can be used either as a list of recommendations to design a specific IS or as an input of a scoring interface.

\subsection{Scorer of Heuristics}

If we want to use the adviser's set of heuristics to evaluate UX, we can carry out the evaluation using this component. It helps UX evaluators in their scoring of heuristics. It should have a control of user profiles to identify if the user is connected to the system as an evaluation administrator or as an evaluator; the interface provides users 
with two parts according to each user profile. The first part is the administration part where administrator should review the heuristics suggested by the adviser, and should choose the evaluators and the severity factors. The second part is the evaluation part where evaluators should score every heuristic. In addition, this component stores all the information to make the extraction of qualitative and quantitative results easier.

\subsection{Results Analyzer}

The last component waits for the extraction of quantitative and qualitative results. In reference to qualitative results we will try to create a list of improvements according to evaluators' observations and heuristics scored incorrectly. They are ones which designers should apply to interactive systems. Apart from qualitative results, we want to obtain quantitative ones because these will try to show the degree of UX that an IS has. If quantitative results are achieved, UX experts will have a standard method or a possible certification to compare evaluations and see which IS provides users with the best experience. Quantitative results will get an objective measure that we cannot achieve with the subjectivity of qualitative results.

\section{Research Already Conducted}

In reference to the evaluation planning, all these ideas started when we had to carry out an evaluation of physical devices called CIPs (Citizen Information Point) [6]. We realised that we could not use some well-known heuristics such as Nielsen's. These were GUI-focused and, they did not cover our evaluation goals and all the features of CIPs. We started researching as much as possible all general usability heuristics defined since 1986 and we detected 16 repeated categories. They are applicable in simple websites or desktop applications [7].

Apart from this review, we achieved a classification of IS components that permitted us to know what parts of the IS should be assessed and to choose the best heuristics for each part [5]. Then, we validated these 16 categories making a comparison between two sets of heuristics to detect which set can find more usability problems and to detect improvements in their definition [8]. And in addition these heuristics might work in semantic applications, now we are working in it. In reference to the results, we have already made an effort to achieve a mathematical formula in usability HE [8], we do not rule out our option but we know that we have to do more research on it.

\section{Current State of Study and Future Research}

Currently, we are working on different areas of this project. The first one is modeling dates to be able to achieve the first version of the adviser. Second, we are working on the design of the second component of our resource: the heuristics scorer component. In addition, while all this work is being done, we are also doing research on all the information needed for our open repository although we know this information about heuristics, facets and new interactive systems can increase (for this reason our repository is an open repository). 
In the future we will primarily work on quantitative results to achieve a statistical or mathematical formula towards UX standardization.

Acknowledgements. The work has been partially supported by Spanish Ministry of Science and Innovation through the Open Platform for Multichannel Content Distribution Management research project (TIN2008-06228) and it has been partially supported by Universitat de Lleida for pre-doctoral fellowship to Llúcia Masip.

\section{References}

1. User Experience design, http://semanticstudios.com/publications/semantics/000029.php

2. Vermeeren, A., Lai-Chong Law, E., Roto, V., Obrist, M., Hoonhout, J., Väänänen-VainioMattila, K.: User Experience Evaluation Methods: Current State and Development Needs. In: Proc. of NordiCHI 2010. ACM, New York (2010)

3. Nielsen, J., Molich, R.: Heuristic evaluation of user interfaces. In: CHI 1990: Proceedings of the SIGCHI Conference on Human Factors in Computing Systems, NY (1990)

4. Johnson, G.I., Clegg, C.W., Ravden, S.J.: Towards a practical method for user interface evaluation. Applied Ergonomics 20(4), 255-260 (1989)

5. Masip, L., Oliva, M., Granollers, T.: Classification of interactive systems components enables planning heuristic evaluation easier. In: HCI International 2011 (accepted paper, 2011)

6. Oliva, M., Masip, L., Granollers, T.: Evaluación de usabilidad y accesibilidad de un conjunto de dispositivos interactivos denominados Puntos de Información Ciudadana. Scire: representación y organización del conocimiento, num 202212 (2010) ISSN: 1135-3716

7. Masip, L., Oliva, M., Granollers, T.: Hacia la semiautomatización de la evaluación heurística: Primer paso, categorización de heurísticas. In: Interacción 2010, Grupo Editorial Garceta, Valencia, September 7-10, pp. 359-368 (2010)

8. Masip, L., Granollers, T., Oliva, M.: A Heuristic Evaluation Experiment To Validate The New Set Of Usability Heuristics. In: Proceedings of 8th International Conference on Information Technology: New Generations, Las Vegas (2011); 978-0-7695-4367-3/11 (C) 2011 IEEE DOI 10.1109/ITNG.2011.82

9. González, M., Masip, L., Granollers, T., Oliva, M.: Quantitative analysis in a heuristic evaluation experiment. Advances in Engineering Software 40(12), 1271-1278 (2009) 